\title{
On the representing number of intersecting families
}

\author{
By \\ M. AlGiNer, P. Erdös and D. Grieserk \\ Herrn Professor Pickert sum 70. Geburtstag in Verehrung gewidmet
}

1. Introduction. One of the best-known results in extremal set theory is the Theorem of Erdös-Ko-Rado [3]:

Suppose $n \geqq 2 k$, and let $M$ be a family of $k$-subsets of an $n$-set $M$ such that any two members of W intersect non-trivially, then $|\mathfrak{M}| \leqq\left(\begin{array}{l}n-1 \\ k-1\end{array}\right)$. Furthermore, the bound can be attained, and the extremal families are precisely the families $\mathfrak{M}_{a}=\{X \ni a: a \in M\}$ for $k \geqq 3$. Many proofs of this result have been given, in addition to the original proof see e. g. $[4,9,10]$. Since all the members of an extremal familiy $M$ have an element in common, we say that $\mathfrak{M}$ has representing number 1.

What if we do not allow the sets of $\mathfrak{M}$ to have an overall nontrivial intersection? How large can then $\mathfrak{M}$ be? The answer to this question has been given by Hilton-Milner [8]

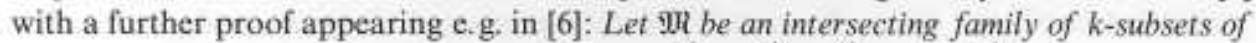
an $n$-set $M$ such that $\bigcap_{x \in m} X=0$, then $|\mathfrak{M}| \leqq\left(\begin{array}{l}n-1 \\ k-1\end{array}\right)-\left(\begin{array}{c}n-k-1 \\ k-1\end{array}\right)+1$ for $n>2 k$. Again the extremal families are characterized. Since the members of $\mathfrak{M}$ are allowed to contain one of two points, but not a single one we say that $\mathfrak{P}$ has representing number 2 .

In this paper we estimate the cardinality of an intersecting family with an arbitrary representing number $r, 1 \leqq r \leqq k$. We first give the relevant definitions. All sets will be assumed to be finite. The collection of all $k$-subsets of a set $M$ will be denoted by $\left(\begin{array}{c}M \\ k\end{array}\right)$. We say that a family $\mathfrak{M}$ is intersecting if any two members of $\mathfrak{M}$ have a non-trivial intersection.

Definition. Let $M$ be a family of sets, and $R$ a single set. $R$ is said to represent $M R$ or be a representing set for $\mathfrak{M}$ if $R \cap X \neq \emptyset$ for all $X \in \mathfrak{M}$. $\mathfrak{M}$ has representing number $r$ if $r$ is the cardinality of a smallest set representing $\mathscr{M}$.

Since an intersecting family $\mathfrak{Q}$ is represented by every one of its members we note that the representing number $r$ of such a family satisfies $r \leqq \min (|X|: X \in \mathfrak{W})$. In particular, if $\mathfrak{M} \subseteq\left(\begin{array}{c}M \\ k\end{array}\right)$ then $1 \leqq r \leqq k$. 
Theorem. Let $n, r, k$ be natural numbers with $1 \leqq r \leqq k \leqq n$. Denote by $g(n ; r, k)$ the maximal cardinality of an intersecting family $M \subseteq\left(\begin{array}{c}M \\ k\end{array}\right)$ of an $n$-set $M$ with representing number $r$. Then there are constants $c_{r, k}, C_{r, k}$ only depending on $r$ and $k$, such that

$$
c_{r, k} n^{k-r} \leqq g(n ; r, k) \leqq C_{r, k} n^{k-r} \text {. }
$$

Sections 2 and 3 are devoted to a proof of this result with a few additional comments appearing in Section 4.

2. Proof of the upper bound. This section establishes the existence of the constant $C_{r, k}$ as spelled out in the statement of the theorem. We divide the proof into a series of lemmas. First we need a definition.

Definition. Let $\mathscr{U}$ be a family of sets and let $u \in \mathbb{N}, u>1$. A $\Delta(u)$-system of $\mathscr{A}$ is a subfamily $\mathfrak{B} \subseteq \mathcal{U}$ such that

(i) $|\mathfrak{B}|=u$,

(ii) any two members of $\mathfrak{B}$ have the same intersection $C . C$ is called the stem of $\mathfrak{B}$.

The following lemma appeared in [2]. The easy proof goes by induction on $a$.

Lemma 1. Let $a, b \in \mathbb{N}, b>1$. Then there exists $a$ smallest number $f(a, b) \in \mathbb{N}$ such that any family of sets $\mathscr{U}$ with $|\mathbb{U}|>f(a, b)$ and $(X \in \mathcal{U} \Rightarrow|X| \leqq a)$ possesses $a$ $\Delta(b)$-system. Furthermore, $f(a, b) \leqq a !(b-1)^{a}$.

Lemma 2. Let $\mathcal{U}$ be a family of sets with $X \in \mathfrak{U} \Rightarrow|X| \leqq k$. Let, further, $\mathfrak{B}$ be a family of sets such that every $X \in \mathfrak{B}$ is a representing set of $\mathscr{Q}$ and satisfies $|X| \leqq b$. If $|\mathfrak{B}|>f(b, k+1)$, then there exists a representing set $Y$ of $2 \mathbb{1}$ with $|Y| \leqq b-1$ and $Y \leqq Z$ for some $Z \in \mathfrak{B}$.

Proof. Let $\left\{Y_{1}, \ldots, Y_{k+1}\right\}$ be a $\Delta(k+1)$-system of $B$ with $\left|Y_{i}\right| \leqq b$ for all $i$ and stem $Y$ (guaranteed by Lemma 1). Then $|Y| \leqq b-1, Y \subseteq Y_{i} \in \mathfrak{B}$. We claim that $Y$ represents $\mathcal{I}$. If, on the contrary, there existed $X \in \mathcal{Q}$ with $X \cap Y=\emptyset$ then $X$ would have to intersect all the disjoint set $Y_{1}-Y, Y_{2}-Y, \ldots, Y_{k+1}-Y$, in contradiction to $|X| \leqq k$.

To facilitate the induction used in the proof of the theorem we introduce the following function.

Definition. Let $n, r, k \in \mathbb{N}$. For $\ell \in \mathbb{N}, \ell \leqq k$ define the functions $h_{\ell}^{\prime}: \mathbb{Q} \rightarrow \mathbb{Q}$

$$
\begin{aligned}
& h_{k}(x)=x \\
& h_{\ell}(x)=\frac{1}{\left(\begin{array}{l}
n-r \\
k-r
\end{array}\right)}(x-f(k, k+1))-\sum_{i=k+1}^{k-1} f(i, k+1) \text { for } \quad t<k .
\end{aligned}
$$

The following facts are immediately verified from the definition. 
Lemma 3. i) $h_{\ell+1}\left(x-h_{\ell}(x)\left(\begin{array}{l}n-r \\ k-r\end{array}\right)\right)=f(\ell+1, k+1)$ for all $x$,

ii) if $x>\left(\begin{array}{l}n-r \\ k-r\end{array}\right) \sum_{i=r}^{k-1} f(i, k+1)+f(k, k+1)$ then $h_{r-1}(x)>0$.

We come to the crux of the proof.

Lemma 4. Let $n, k, r$ and $M, \mathfrak{M}$ be given as in the statement of the theorem. For $a$ subfamily $\mathfrak{N} \subseteq \mathfrak{M}$ and $\ell \leqq k$ let

$$
\begin{aligned}
& \mathbb{M}_{c}=\left\{X \subseteq M: X \text { represents } \mathbb{M},|X| \leqq \ell \text { and there exists } Y \in \mathbb{P R}^{\prime}\right. \text { with } \\
& X \subseteq Y\} \text {. }
\end{aligned}
$$

Then $\left|\mathfrak{M} \mathcal{R}_{\ell}\right| \geqq h_{\ell}(|\mathfrak{M}|)$.

P r o of. We use downward induction on $\ell$. For $\ell=k$ we have $\mathfrak{M}_{\alpha} \supseteq \mathbb{M}^{\prime}$ and thus $\left|\mathfrak{M}_{k}^{\prime}\right| \geqq h_{k}\left(\left|\mathfrak{M}^{\prime}\right|\right)=\left|\mathfrak{P}^{\prime}\right|$. Suppose we already know that $\left|\mathfrak{M}_{c+1}\right| \geqq h_{c+1}\left(\left|\mathfrak{M}^{\prime}\right|\right)$ holds for all subfamilies $\mathfrak{M}^{\prime} \subseteq \mathfrak{M}$. We determine step by step distinct sets $X_{1}, X_{2}, \ldots, X_{2} \in \mathfrak{W}$, with $\alpha=\max \left(0,\left[h_{\ell}\left(\left|\mathbb{M}^{\prime}\right|\right)\right]\right)$. Let $\alpha>0$ and $1 \leqq \beta \leqq \alpha$. Suppose we have already found sets $X_{1}, X_{2}, \ldots, X_{\beta-1} \in \mathfrak{M}$, Set

$$
\begin{aligned}
\mathfrak{M}^{N} & =\left\{X \in \mathfrak{M}^{\prime}: X \supseteqq X_{i} \text { for some } i, 1 \leqq i \leqq \beta-1\right\} \\
\mathfrak{\mathbb { R }} & =\mathfrak{M}^{\prime}-\mathfrak{M}^{\prime} .
\end{aligned}
$$

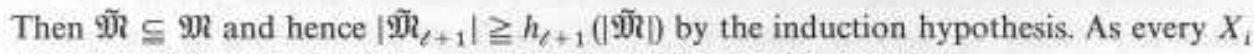
represents $\mathfrak{M}$ we have $\left|X_{i}\right| \geqq r$ by the assumption on $9 R$, and thus

$$
\left|\left\{X \leqq M: X \supseteq X_{i}\right\}\right| \leqq\left(\begin{array}{l}
n-r \\
k-r
\end{array}\right) \quad(i=1, \ldots, \beta-1) .
$$

From this we infer

$$
\begin{aligned}
|\mathfrak{\mathscr { W }}| & =\left|\mathfrak{M}^{\prime}\right|-\left|\mathbb{M}^{\prime \prime}\right| \\
& \geqq\left|\mathfrak{M}^{\prime}\right|-(\beta-1)\left(\begin{array}{l}
n-r \\
k-r
\end{array}\right) \\
& \geqq|\mathfrak{M}|-(\alpha-1)\left(\begin{array}{l}
n-r \\
k-r
\end{array}\right) \\
& >\left|\mathfrak{M}^{\prime}\right|-h_{\ell}\left(\left|\mathfrak{W}^{\prime}\right|\right)\left(\begin{array}{l}
n-r \\
k-r
\end{array}\right) .
\end{aligned}
$$

Since $h_{t+1}$ is strictly increasing we conclude from Lemma 3 (i)

$$
\left|\mathfrak{M} h_{\ell+1}\right| \geqq h_{\ell+1}(|\mathfrak{\mathscr { D }}|)>f(\ell+1, k+1) .
$$

Now Lemma 2 applied to $\mathscr{A}=\mathfrak{M}, \mathfrak{B}=\mathfrak{9} \Re_{\ell+1}$ implies the existence of a set $X_{\beta}$ with $\left|X_{\beta}\right| \leqq \ell$ representing $\mathfrak{W}$ and of $Y \in \mathscr{\mathscr { R }}_{\ell+1}$ with $X_{\beta} \subseteq Y$. $Y$ is, in turn, contained in a set $Z \in \tilde{\mathcal{R}}, Y \subseteq Z$, by the definition of $\widetilde{\mathfrak{M}}_{k+1}$. In summary, $X_{j} \subseteq Z \in \mathscr{\mathfrak { M }}_{\mathcal{R}} \subseteq \mathfrak{M}$. Hence $X_{\beta} \in \mathfrak{M}_{r}$ and $X_{\beta}$ must be distinct from all sets $X_{1}, \ldots, X_{\theta-1}$ since $X_{\beta}=X_{i}$ would imply $Z \in \mathfrak{M}^{\prime \prime}=\mathfrak{M}^{\prime}-\mathfrak{Q}$, whereas $Z \in \mathscr{M}$. 
Proof of the upper bound. Suppose, on the contrary, there is no such constant $C_{r, k}$. Then there are $n, M$ and a family $m$ satisfying the assumptions of the theorem with

$$
|\mathfrak{M}|>\left(\begin{array}{l}
n-r \\
k-r
\end{array}\right) \sum_{i=r}^{k-1} f(i, k+1)+f(k, k+1) .
$$

Applying Lemma 4 with $\mathfrak{M} \tau^{\prime}=\mathfrak{M}$ and $\ell=r-1$, we conclude $\left|\mathbb{R}_{r-1}\right| \geqq h_{r-1}(|\mathfrak{M}|)$ and thus $|\mathfrak{M},-1|>0$ by Lemma 3 (ii). But this contradicts the fact that $\mathfrak{M}$ cannot be represented by a set of cardinality less than $r$, and the proof is complete.

From the inequality $\left(^{*}\right)$ and Lemma 1 we obtain the following estimate of $C_{r, k}$.

Corollary. For given $n, r, k$ and $M, \mathfrak{M}$ as in the statement of the theorem we have

$$
|\mathfrak{M}| \leqq\left(\sum_{i=r}^{k} i ! k^{i}\right) n^{k-r} .
$$

3. Proof of the lower bound. Let $r$ and $k$ be given. The Erdös-Ko-Rado Theorem states $g(n ; 1, k)=\left(\begin{array}{l}n-1 \\ k-1\end{array}\right)$ for $n \geqq 2 k$, hence $c_{1, k}$ exists. For $r>1$ we use a generalization of the construction in [1] which includes the optimal family of the Hilton-Milner Theorem [8] for $r=2$ and the one given by FrankI [5] for $r=3$ as special cases.

Assume $n \geqq k+(k-1)+\cdots+(k-r+2)+1$. Choose pairwise disjoint sets $\mathrm{S}_{i}(i=0, \ldots, r-2)$ with $\left|S_{i}\right|=k-i$, a subset $T \subseteq S_{0}$ with $|T|=r-1$ and an element $x \notin \bigcup S_{i}$. Denote by $\mathfrak{M}_{i}$ the family

$$
\begin{aligned}
& \mathfrak{M}_{i}=\left\{X: X \supseteqq S_{i},\left|X \cap S_{j}\right|=1 \text { for } 1 \leqq j<i,|X \cap T|=1\right\} \\
& (i=1, \ldots, r-2),
\end{aligned}
$$

and by $\mathfrak{M}_{x}$ the family

$$
\mathbb{M}_{x}=\left\{X:|X|=k, x \in X, X \cap S_{i} \neq \emptyset \text { for all } i\right\} \cup\{X:|X|=k, x \cup T \subseteq X\} .
$$

The family $\mathfrak{M}=\bigcup_{i=1}^{-2} \mathfrak{M}_{i} \cup \mathfrak{M}_{*} \cup\left\{S_{0}\right\}$ is intersecting, has $T \cup x$ as representing set, and it is readily seen that no smaller set can represent $\mathfrak{M}$. Since the second part of $\mathfrak{M} \mathbb{R}_{x}$ contains already $\left(\begin{array}{l}n-r \\ k-r\end{array}\right)$ sets, the existence of $c_{r, k}$ is established.

4. Families with representing number $k$. As mentioned before, the precise value of $g(n ; 1, k)$ and $g(n ; 2, k)$ is known whereas the family $\mathfrak{M}$ of the previous section was shown to be optimal in [5] for $r=3$ and $n \geqq n_{0}(k)$. Let us go to the other end and consider $g(n ; k, k)$.

The theorem says in this case that $g(n ; k, k)$ is independent of $n$ for $n \geqq n_{0}(k)$, so we denote it shortly by $g(k)$.

The corollary in Sect. 2 gives $g(k) \leqq k ! k^{k}$, and it was shown in [1] that, in fact, $g(k) \leqq k^{k}$. To gain further insight into $g(k)$ we observe that any maximal family 
$\mathfrak{W} \subseteq\left(\begin{array}{c}M \\ k\end{array}\right)$ with representing number $k$ must include all representing sets of $\mathfrak{N}$ of size $k$. This, in turn, immediately yields the following alternate characterization.

Proposition. Let $\mathfrak{M} \subseteq\left(\begin{array}{c}M \\ k\end{array}\right)$ be an intersecting family. Then the following condi-
tions are equivalent:

i) $\mathfrak{M}$ is maximal with representing number $k$.

ii) $\mathfrak{M}$ is maximal with respect to the condition that to every $X \in \mathfrak{M}, x \in X$ there exists $Y \in \mathfrak{M}$ with $X \cap Y=\{x\}$,

The construction of Erdös and Lovász in [1] yields $g(k) \geqq k ! \sum_{i=1}^{k} \frac{1}{i !}$, and thus $g(k) \geqq(e-1) k !$ for $k \rightarrow \infty$. For small $k$, we have $g(1)=1, g(2)=3$. Using the preceding proposition it can be easily shown that $g(3)=10$ and, with a little more work, $g(4)=41$ which was also found in [7]. Hence for these values, the construction in [1] is optimal, and it is quite plausible that optimality always holds.

Two interesting questions come to mind: First, improve the bounds on $g(k)$, and, secondly, estimate the threshold value $n_{0}(k)$.

A c k now ledgement. The authors are grateful for some very useful comments by Z. Füredi who independently proved our main theorem.

\section{References}

[1] P. Erdös and L. Lovàsz, Problems and results on 3-chromatic hypergraphs and some related questions. In: Finite and Infinite Sets (A. Hainal et al,, eds.). Proc. Coll. Math. Soc. J. Bolyai 10, $609-627$ (1974).

[2] P. ERDös and R. RADO, Intersection theorems for systems of sets. J. London Math. Soc. 35, $85-90(1960)$.

[3] P. ERDós, C. Ko and R. RADO, Intersection theorems for systems of finite sets. Quart. J. Oxford Ser, 12, 313-320 (1961).

[4] D. DAYkın, Erdös-Ko-Rado from Kruskal-Katona. J. Combin. Theory (A) 17, 254-255 (1974).

[5] P. FrANKL, On intersecting families of finite sets. Bull. Austral. Math. Soc. 20, 363-372 (1980).

[6] P. FrankL. and Z. FürIDI, Non-trivial intersecting families. To appear.

[7] D. Hanson and B. ToFt, On the maximum number of vertices in $m$-uniform cliques, Ars Combin. (A) 16, 205-216 (1983).

[8] P. Hilton and E. Mriner, Some intersection theorems for systems of finite sets. Quart. J. Math. Oxford (2), 18, 369-384 (1967).

[9] G. Katona, A simple proof of the Erdös-Ko-Rado theorem. J. Combin. Theory 13, 183-184 (1972).

[10] R. Wuson, The exact bound in the Erdös-Ko-Rado theorem. Combinatorica $(2-3) 4,247-257$ (1984).

Eingegangen am 28, 8.1986

Anschriften der Autoren:

M. Aigner und D. Grieser

II. Math. Institut

Freic Universităt Berlin,

Arnimallee 3

D-1000 Berlin 33
P. Erdös

Math. Institut

Ungarische Akademie der Wissenschaften

Realtánoda u. 13-15

H-1053 Budapest 